\title{
A Cost-Effective Technology-Assisted Communication for Paralyzed People using Eye Blink - An Experimental Approach
}

\author{
Qurban Memon $^{\mathrm{a}}$, Naser Alteneiji ${ }^{\mathrm{b}}$, Mugbil El Sayed ${ }^{\mathrm{b}}$, Mohamed Tarig ${ }^{\mathrm{b}}$, Abdirahim Hassan $^{\mathrm{b}}$ \\ ${ }^{a}$ Associate Professor, College of Engineering, UAE University. \\ ${ }^{\mathrm{b}}$ Research student,_College of Engineering, UAE University.
}

Correspondence Author: Qurban Memon, Associate Professor, College of Engineering, UAE University,

Received date: 18 June 2019, Accepted date: 26 September 2019, Online date: 29 September 2019

Copyright: (C) 2019 Qurban Memon et al, This is an open-access article distributed under the terms of the Creative Commons Attribution License, which permits unrestricted use, distribution, and reproduction in any medium, provided the original author and source are credited.

\begin{abstract}
Over decades, a number of solutions have been placed in practice to help physically challenged person to communicate with environment. The solutions refer to devices that help manage living environment such as opening a window, adjusting AC, controlling TV or turning lights off and on. One such solution is in the form of keyboard, where a care giver, for example a nurse, lets paralyzed person's eyeball scan the keyboard to select options on it to understand his/her needs. Currently, technology-assisted communication is possible, but is limited and at a higher cost. In this research, a customized soft keyboard and cost-effective eye blink communication system are presented to connect to an electric movable bed to select a comfortable bed position for the paralyzed without help of a nurse or a care-giver. An experimental setup is built and results are presented with accuracy of $\mathbf{9 5 \%}$, and with an operational time of less than a second. It is also shown that the built system can be easily modified and extended to facilitate the paralyzed person for various other movable machines, for example, wheelchair or remotely-controlled machines in home environment, or even at driving, etc. The practical constraints are also discussed.
\end{abstract}

Keywords: Eyeblink, Technology-Assisted Communication, Rehabilitation, Scanning keyboard.

\section{INTRODUCTION}

Physical challenges emanate from problems and diseases such as spinal cord injuries due to accidents resulting in paralysis of various forms, etc. In some cases, these injuries force the patient to remain in bed or on wheelchair for life, with limited mobility possible only with the help of care-giver. Such problems bring several challenges especially if person cannot communicate and/or move body muscles. It can be envisioned easily that the human audio-visual system connected to a computing device and interfaced to Internet can provide:

a) eye-tracking solutions for persons suffering from some form of paralysis.

b) phone-apps to increase independence.

c) adapted-communication devices can be built to live safely.

d) home-automation devices can be built to provide assistive technology-based solutions to paralyzed persons.

Over a number of decades, different solutions have been in practice to help the physically challenged person to communicate with other people. Rehabilitation centres, both public and private, have propped up and provided care to physically challenged people, but lack of self-communication in some way has increased problems of this growing community (Balckstone, S., et al, 2015). One traditional approach that has existed quite a time now, is the partner-assisted scanning (Online Assistive Technology Forum, 2017), where a nurse uses a keyboard in front of the paralyzed person to scan his/her eyeball in selecting different characters or words to enable communication. A number of research works can be found be in literature, where authors have attempted to facilitate the living of the paralyzed by developing algorithms and tools. For example, the authors (Praveenkumar, B., Mahendrakan, K., 2014) monitor to detect driver's drowsiness in order to deploy preventive measures to avoid an accident. The measures include weighing and deploying controls using infrared sensor transmit these rays to driver's eyes. Similarly, authors (Z. A. Haq and Z. Hasan, 2016) predict cognitive load and fatigue in drivers using eye-blink rate detection, with a success rate of $87 \%$. The authors claim that it can greatly reduce the amount of fatigue related to accidents so that preventing measures can be alerted. The authors (Aleksandra Królak, A., Strumiłło, P., 2011) build an eye blink detection solution as an alternative means to interact with computers and machines. In another work (Rihana S., Damien P., Moujaess T., 2013), the authors develop EEG- 
based eye blink detection for brain-computer interface to be used in neurorehabilitation application. For further reading, the readers are referred to recent works (Young-Joo, H., et al., 2018; T. N. Bhaskar, et al, 2003; Al-Kassim, Z., Memon, Q., 2017; Pasarica, A., et al, 2016).

In this research, we aim to build low-cost technology-assisted eye blink detection using custom-designed keyboard interfaced to, for example, a movable bed. The interface interprets these eye blinks as control commands. With an appropriate design of the keyboard, the person on bed, for example, can also automatically select an option on the keyboard to communicate his choice to open or close curtain, switch on/off TV, moving bed to another position, etc. The paper is structured as follows. In next section, we present the proposed approach for eye blink detection to be interfaced with a movable bed. Following this, another section presents simulation and experimental results, followed by conclusions.

\section{PROPOSED APPROACH}

In this section, we present the approach to building an eye blink detection to be used as a technology-assisted communication tool for the paralyzed. As a first step, camera positioning using a laptop computer was conducted. For writing code, initially face detection was completed based on Viola-Jones classifier (Viola, P., Jones, M., 2004) to determine the area around eyes. This area turns out to be our region of interest (ROI). This region of interest is enhanced to offset any possible shadow, lighting, or makeup effects. This can be effectively be achieved through multiplying ROI pixels with a value gamma $(\gamma)$, as:

$\gamma=\left\{\begin{array}{ll}1-\alpha, & \alpha>0 \\ \frac{1}{1+\alpha}, & \alpha \leq 0\end{array}\right\}$

where $\alpha$ is a user-defined value. Next, the color pixels are converted to grey level in order to retrieve its luminance value. The approach used here is based on differentiating between eyelashes, pupil and eye boundaries as one region and skin color as another. This approach is called as histogram backpropagation. This approach uses skin color to differentiate between open and closed eyes. In other words, skin color in closed eye state will have more count of skin color pixels as opposed open eyes. This higher value in eye region represents objection location more likely.

In order to proceed, the grey level values are further enhanced before converting to black and white. The resulting black pixels represent eyelashes, pupil, and eye boundaries, whereas the white pixels represent skin color. Since the values are continuous, we used a value of 0.25 to differentiate between white and black pixels, i.e., the pixel values above 0.25 count to region 1 (white) and below to 0 (black). In order to make the decision for open or closed eyes, these pixel values are counted. As can be judged easily, in case of open eyes, the pixels related to pupil will also map to black. Thus, for open eyes, the black pixels will be more than white area pixels. In the form an equation, this decision can be formulated as:

Skin Percentage $=($ White Area Pixels in ROI) / (ROI Pixels $) \times 100$

Using trial and error, a threshold of $90 \%$ was determined to indicate skin percentage when eyes are closed, and open when this percentage falls below $90 \%$.

Next step in our approach was to link this eye blink detection to software to work with a screen keyboard. A set of most commonly needed symbols were used as rectangular layout of keys to be selected in coordination with eye blink. This keyboard layout is shown in Figure 1:

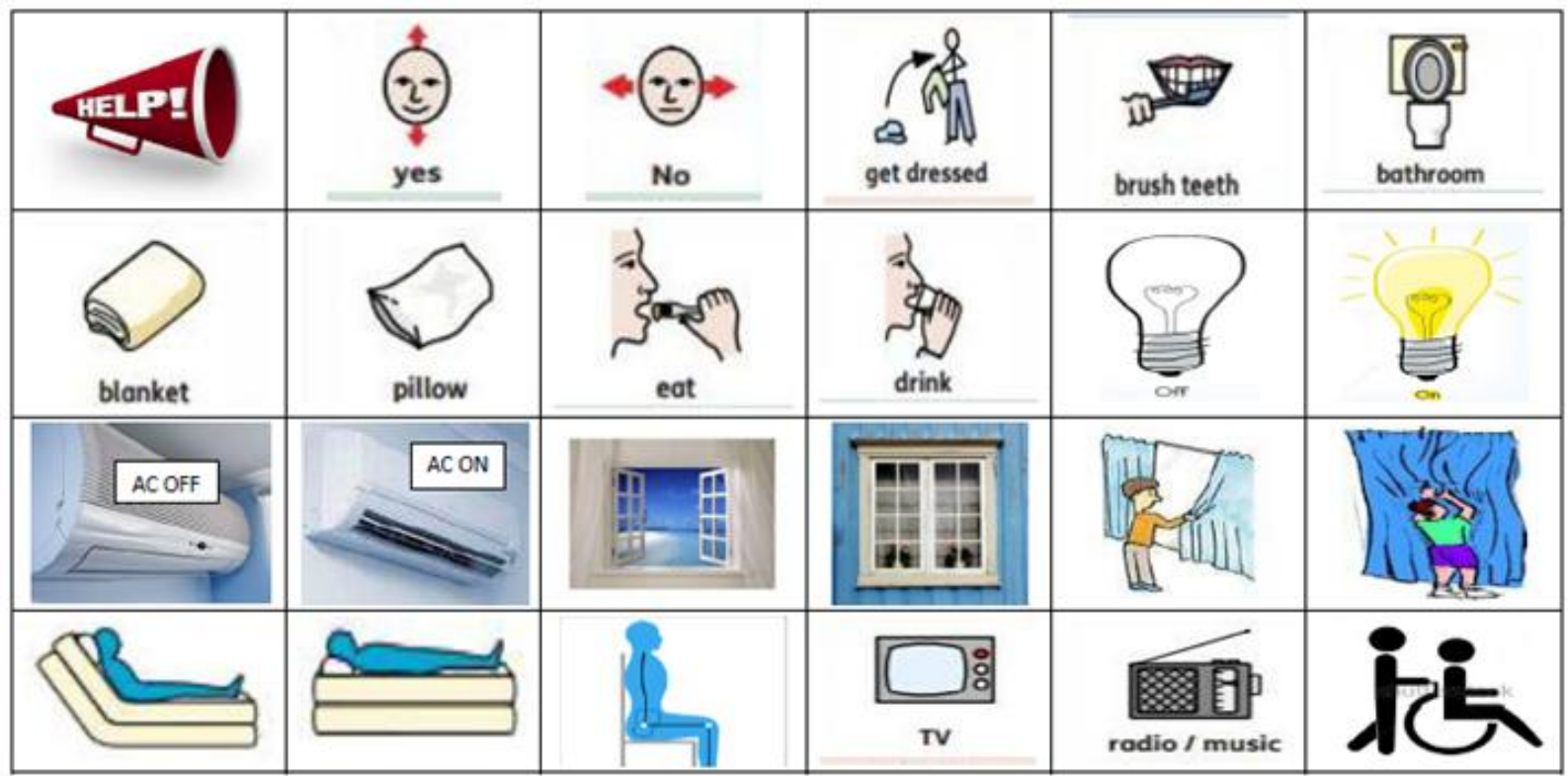

Figure 1: Rectangular Layout for a soft keyboard 
Each picture symbol in the keyboard is called a key and represents a needed service by the paralyzed. The eye blink detection will be considered as a selection of a particular key. The system starts with top-left key (blinking with green color background). It, then, scrolls each key rightwards with a pause of 2 seconds between keys. During this pause, the highlighted key blinks with green background color. The start-key left or right scroll and pause can be selected/adjusted based on the choice and per requirements of the person. The rows are selected in sequence and at the end of last (bottom-right) key, the system re-starts at top-left key. The operational flow of the system linking eye blink and hardware/machine to control is depicted in Figure 2. It shows how software program links to hardware and works as technology-enabled control. Theoretically, a number of devices can be interfaced, as shown in Figure 2, but in experimental setup only one device is picked to test the performance of the proposed solution.

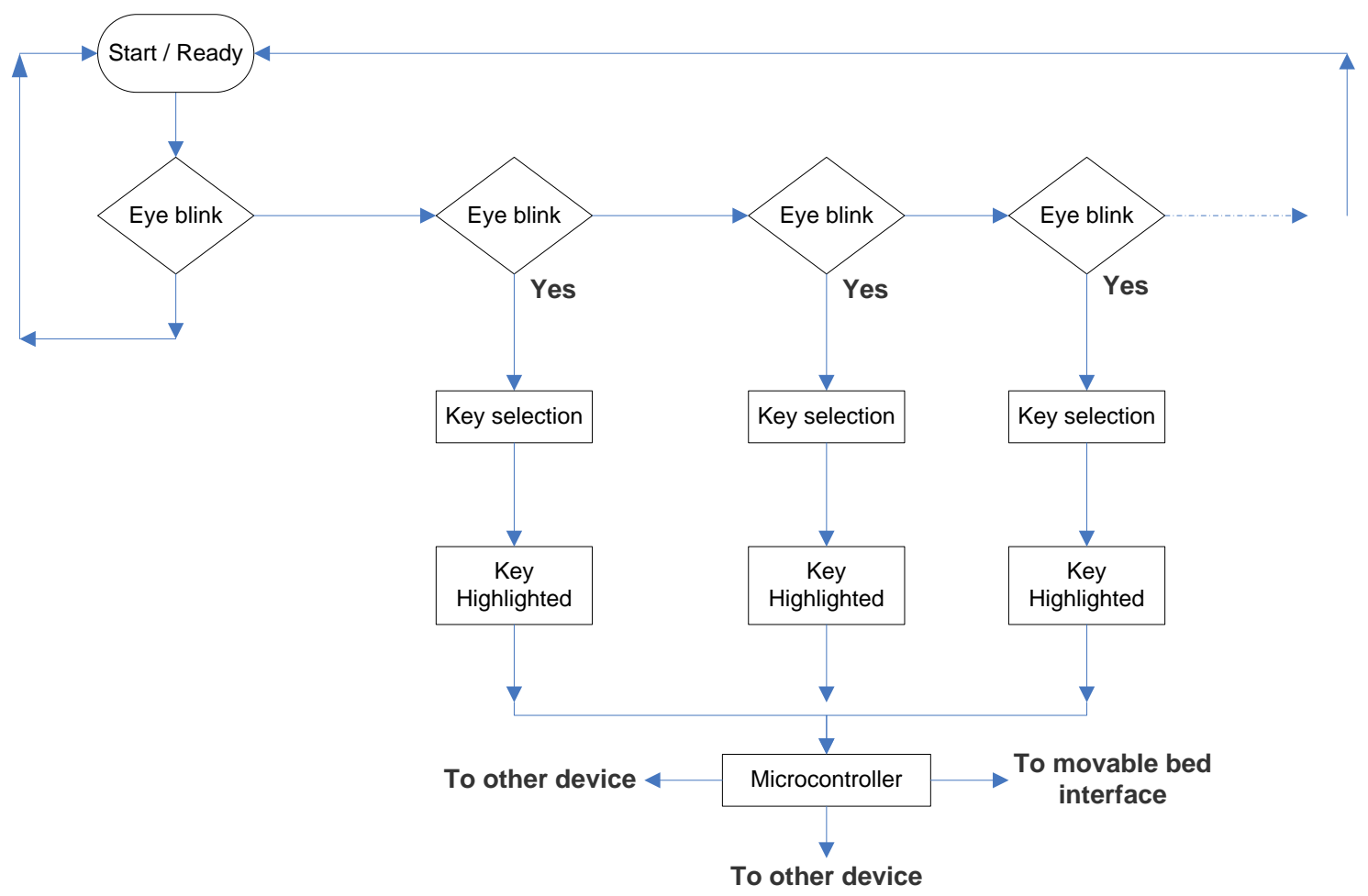

Figure 2: Operational flow of the system

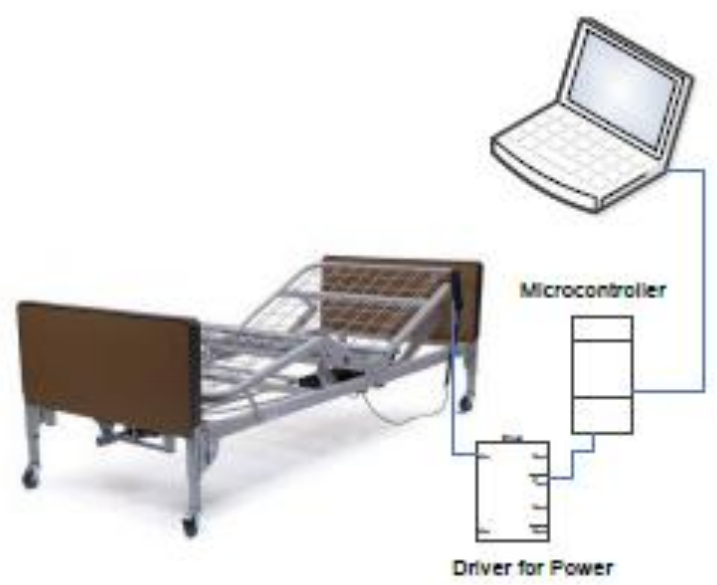

Figure 3: Experimental Setup

\section{EXPERIMENTAL RESULTS}

In order to test the performance of the system (as illustrated in Figure 2), an electric movable bed (with various head, knee and height adjustments) as shown in Figure 3 was connected through the microcontroller to laptop computer. The driver was selected based on the power requirements of the movable bed. Based on approach discussed in section 2, a code was written in Matlab to detect open or closed eyes individually to check the performance of the system. Two checks were planned to see success rate of 
the program. In the first case, both eyes once open, were kept open to see if it detects open eyes. Fifty trials were run on four persons (with about 12 trials per persons). The system succeeded in detecting open eyes for 47 trials out of 50. The net success rate, in this case, turned out to be $94 \%$. For second case, once eyes were open, were later closed to detect blink. The same number of trials were repeated, with a success rate of $96 \%$. The overall success rate of the system is $95 \%$. The individual results pertaining to each person, for each case, are displayed in Table 1, as a ratio of successful results and overall trials. The overall success rate exceeds the rate reported in Divjak, M., Bischof, H., 2009; and Heishman, R., Duric, Z., 2007. The total operational time that includes code run time and communication was found to be around 650 msec. Using available current technologies, this code can be transported to any platform including Android. Additionally, a Wi-Fi-enabled microcontroller (Online Arduino WiFi, 2019) can be connected to movable bed to be controlled from a smartphone. Therefore, a cost-effective solution can be built easily using current technologies. There are, however, constraints in this experimental setup: (i) the background of environment or personal clothing should not be of skin color (ii) person should sit in front of keyboard screen and look straight into the camera (iii) both eyeballs should be aligned (iv) camera needs to be calibrated for the setup before testing (Al-Kassim, Z., Memon, Q., 2015).

Table (1): Results

\begin{tabular}{|c|c|c|c|c|c|c|c|}
\hline Test & Person 1 & Person 2 & Person 3 & Person 4 & Average & Overall Success Rate & Total time \\
\hline 1 & $12 / 13$ & $12 / 12$ & $11 / 13$ & $12 / 12$ & $47 / 50=94 \%$ & \multirow{2}{*}{$95 \%$} & $0.14+0.5=0.64$ \\
\cline { 1 - 5 } 2 & $12 / 12$ & $12 / 13$ & $12 / 12$ & $12 / 13$ & $48 / 50=96 \%$ & sec \\
\hline
\end{tabular}

\section{DISCUSSION AND CONCLUSIONS}

A technology-enabled cost-effective communication solution to be used by paralyzed persons was presented with performance accuracy of $95 \%$. Though, a number of eye blink methods and implementations exist in literature, but in this research, customization based on two criteria was set: total operational time (that includes code run-time and communication with movable bed), and the cost that depends on webcam (already available in laptops), microcontroller and its power driver. Additionally, if laptop is connected to Internet, Internet technology can be used to monitor the well-being of the paralyzed person. The soft keyboard presented in this work can be adjusted or modified based on the needs of the environment. The scroll pause of 2 seconds between keys can also be changed based on run-time limits of the program and communication time. Furthermore, this type of communication solution can only be applied to those class of paralyzed persons, who have not suffered vision loss due to paralysis. From communication to environmental control, the proposed solution provides independence from care-giver or family members.

The studies conducted in works (Bentivoglio AR, et al., 1997; Abusharha AA, 2017) measured blink rate variations in various volunteers performing different tasks such as reading a passage, resting, and talking. Mean blink rate at rest was found to be 17 blinks/min. The most common blink rate was conversation followed by rest and then reading (in decreasing order) observed in 101 out of 150 subjects. These studies provide a normal reference for the analysis of the blink rate. In another study (Simone, B., et al., 2011) a normal blink lasts, as average, about 1/10th of a second. Based on these studies, thus, the paralyzed person using this device (in resting mode) can be trained in order to differentiate his/her blinking duration from a normal blink.

\section{ACKNOWLEDGEMENTS}

The authors would like to thank UAE University for SURE+ grant number G00002431 (as a financial support) to undertake this study and research.

\section{REFERENCES}

Abusharha AA, "Changes in blink rate and ocular symptoms during different reading tasks," Clinical Optometry, Vol. 9 pp. 133 138,2017

Aleksandra Królak, A., Strumiłło, P., "Eye-blink detection system for human-computer interaction," Universal Access in the Information Society, 2011, 11(4):1-11

Al-Kassim, Z., Memon, Q., "Designing a low-cost eyeball tracking keyboard for paralyzed people," Computers and Electrical Engineering, Volume 58, February 2017, Pages 20-29

Al-Kassim, Z., Memon, Q., "Experimental Analysis of Calibration Techniques for Eye Tracking," International Conference on Computing and Informatics, Istanbul, 2015, pp. 142-151

Bentivoglio AR, et al., "Analysis of blink rate patterns in normal subjects," Movement Disorders, Vol., 12(6), pp. 1028-1034, 1997

Divjak, M., Bischof, H., (2009). Eye-blink based fatigue detection for prevention of Computer Vision Syndrome, In Proc. Conf. on Machine Vision Applications (MVA), pp. 350-353

Heishman, R., Duric, Z., (2007). Using Image Flow to Detect Eye-blinks in Color Videos, IEEE Workshop on Applications of Computer Vision, p. 52

http://www.apparelyzed.com/forums/forum/62-assistive-technology/ Accessed online on March 22, 2017 
https://www.arduino.cc/en/Guide/ArduinoUnoWiFi, accessed online on April 10, 2019.

Pasarica, A., Bozomitu, R., Cehan, V., Rotariu, C., "Eye Blinking Detection to Perform Selection for an Eye Tracking System Used in Assistive Technology," IEEE International Symposium for Design and Technology in Electronic Packaging, 2016, 212-215, Oradea

Praveenkumar, B., Mahendrakan, K., "Prevention of Accident Due To Drowsy By Using Eye Blink, "International Journal of Innovative Research in Science, Engineering and Technology, Vol. 3, Issue 5, May 2014

Rihana S., Damien P., Moujaess T. (2013), EEG-Eye Blink Detection System for Brain Computer Interface. In: Pons J., Torricelli D., Pajaro M. (eds) Converging Clinical and Engineering Research on Neurorehabilitation. Biosystems \& Biorobotics, Vol 1, Springer, Berlin, Heidelberg

Sarah Blackstone, David Beukelman, Kathryn Yorkston, Patient-Provider Communication: Roles for Speech-Language Pathologists and Other Healthcare professionals, Plural Publishers, 2015.

Simone, B., et al., "Driver workload and eye blink duration," Transportation Research Part F: Traffic Psychology and Behavior, Vol. 14(3), pp.199-208, 2011

T. N. Bhaskar, Foo Tun Keat, S. Ranganath and Y. V. Venkatesh, "Blink detection and eye tracking for eye localization," International Conference on Convergent Technologies for Asia-Pacific Region, Bangalore, India, 2003, Vol.2, pp. 821-824

Viola, P., Jones, M.,'Robust Real-time Object Detection," International Journal of Computer Vision, 57(2), 137-154, 2004.

Young-Joo, H., Wooseong, K., and Joon-Sang, P., "Efficient Eye-Blinking Detection on Smartphones: A Hybrid Approach Based on Deep Learning," Mobile Information Systems, vol. 2018, Article ID 6929762, 2018. https://doi.org/10.1155/2018/6929762.

Z. A. Haq and Z. Hasan, "Eye-blink rate detection for fatigue determination," International Conference on Information Processing, Delhi, 2016, pp. 1-5. 